\title{
Excellent Response of Dermatophytoma and Nail Splitting to Nail Plate Debridement Plus Topical Ciclopirox Olamine 1\% Solution
}

\author{
Hamed Mohamed Abdo \\ Department of Dermatology, Faculty of Medicine, Al Azhar University, Cairo, Egypt
}

\section{Established Facts}

- Dermatophytoma is a special form of onychomycosis which is often resistant to traditional therapy.

- Oral antifungal treatment alone is not enough to treat the condition, and either chemical or physical debridement is essential to eliminate dermatophytoma.

- Ciclopirox nail lacquer $8 \%$ solution has been used to treat some milder forms of onychomycosis with varied efficacy.

\section{Novel Insights}

- Avoiding oral therapy, the current case represents a complete clearance of dermatophytoma and nail splitting with physical nail debridement plus local therapy within 5 months.

- Ciclopirox olamine $1 \%$ solution can be effectively used to treat some forms of onychomycosis when combined with nail plate debridement.

\section{Keywords}

Onychomycosis - Dermatophytoma - Trichophyton rubrum . Nail splitting · Nail debridement · Topical 1\% Ciclopirox olamine

\section{Abstract \\ Onychomycosis often presents as thickened, discolored nails. Usually, one or both great toenails are affected. Even- tually, the nail plate becomes friable and may split and break up, often due to trauma or invasion of the plate by dermato- phytes that have keratolytic properties. Dermatophytoma is a unique feature of onychomycosis that occurred by abun-}

dant fungal filaments and spores forming a fungal ball under the nail plate. It is often refractory to traditional therapy. Based on the clinical and mycological examination, a case of onychomycosis in a 45-year-old woman presented as dermatophytoma with longitudinal nail splitting caused by Trichophyton rubrum is presented. The case was successfully treated with nail plate debridement plus topical ciclopirox olamine $1 \%$ solution. Nail debridement was performed using a 15-scalpel blade to remove the affected nail portion with the underlying subungual debris to enhance the effect of topical ciclopirox $1 \%$ solution which was then applied 3 times daily to the debrided area. Follow-up visits with nail photography were planned every 2 weeks after the proce- karger@karger.com

www.karger.com/sad

(c) 2020 S. Karger AG, Basel

Karger ${ }^{\prime}=$
Hamed Mohamed Abdo

Department of Dermatology, Faculty of Medicine, Al Azhar University

Al-Hussein University Hospital, Gawhar Al Qaed Street, Al Darrasa

Cairo 11633 (Egypt)

hamed392@yahoo.com 
dure to check treatment progress. A complete clinical resolution was achieved after 5 months. This treatment option can be advocated for similar nail conditions avoiding unnecessary and expensive lines of treatment. @2020 S. Karger AG, Basel

\section{Introduction}

Onychomycosis is a difficult-to-treat fungal infection of the nail plate or bed of toenails and/or fingernails leading to a hard, brittle, and uneven nail surface [1]. Oral and topical drug delivery systems are the most desirable in treating onychomycosis, but their efficacy is low, resulting in a relapse rate of $25-30 \%$. Topical therapy improves patient compliance and reduces the cost of treatment. However, due to poor penetration of topical therapy across the nail plate, research is focused on different chemical, mechanical, and physical methods to improve drug delivery [2].

Dermatophytoma, primarily caused by dermatophytes, represents a difficult-to-treat complication of onychomycosis. Dermatophytomas appear as yellow or white streaks or patches and represent a dense concentration of fungal hyphae encased in a layer of biofilm within or under the nail plate. They are less responsive to systemic antifungal therapy [3]. An effective therapeutic agent must be able to diffuse through the nail plate and remain at high enough antimicrobial concentrations in the subungual space [4]. In this respect, dermatophytoma is generally treated with a combination approach, often including both surgical and pharmacologic intervention for improved outcomes [5].

\section{Case Report}

A 45-year-old housewife presented with a 3-month history of nail lesion affecting only the right toenail. On examination, the involved nail showed a longitudinal band of yellow nail plate discoloration, about $5 \mathrm{~mm}$ wide and $10 \mathrm{~mm}$ long, extending from the free edge toward the nail root sparing about $2 \mathrm{~mm}$ distal to the proximal nail fold. Within the yellow band, there was a longitudinal nail splitting wider at its distal end. The remaining part of the nail, and other toenail and fingernails were normal. Nail folds and periungual skin were also normal (Fig. 1a). No history of noticeable nail trauma, treatment, or relevant drug intake. No family history of similar conditions. She had no other skin, hair, teeth, or mucous membrane disorders and was generally in good physical health. Routine blood tests were within normal limits. Mycology work up revealed positive direct microscopy and fungal culturerecovered Trichophyton rubrum.

Based on the clinical and mycological examination, a case of dermatophytoma in a 45-year-old woman with nail splitting is pre-

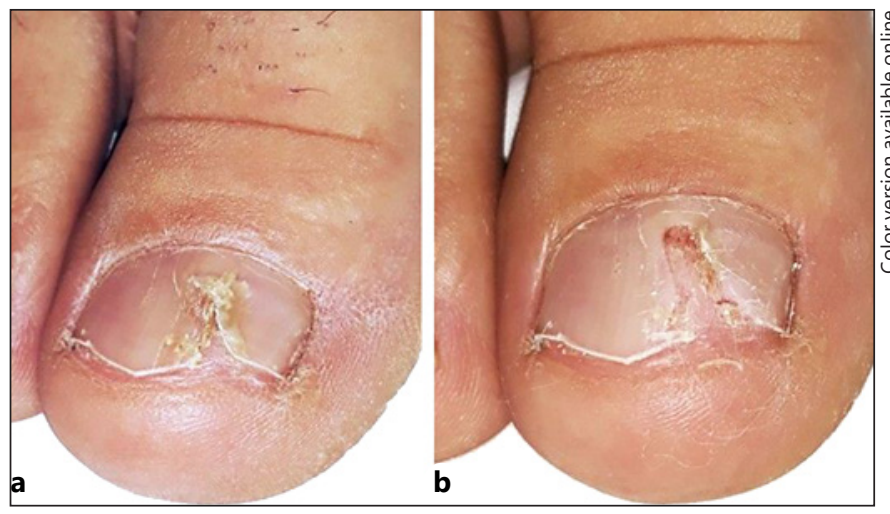

Fig. 1. Nail at 1st visit. a Dermatophytoma with nail splitting. b Nail after debridement.

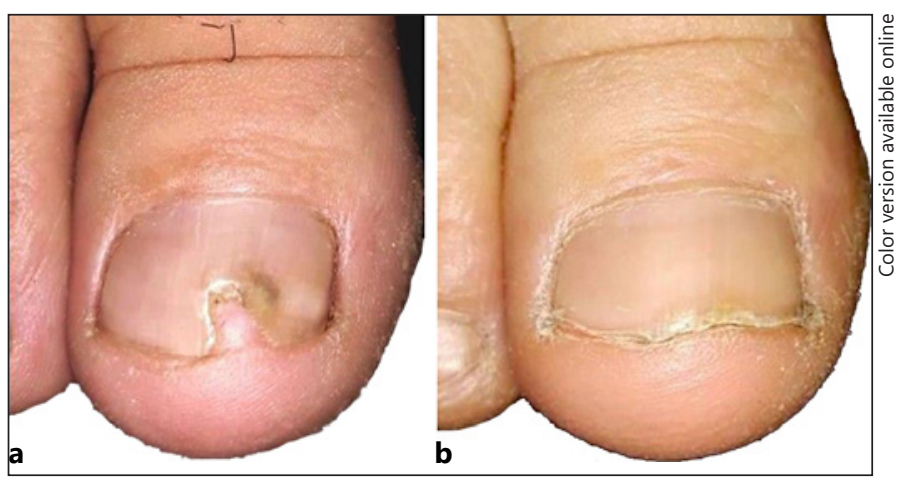

Fig. 2. Follow-up. The same nail after 3 months (a) and 5 months (b) with a complete clinical cure.

sented. A simple treatment plan was issued and included nail plate debridement plus topical ciclopirox olamine $1 \%$ solution. A 15 -scalpel blade was used to remove the affected nail portion with the underlying subungual debris (Fig. 1b) to enhance the effect of topical ciclopirox $1 \%$ solution which was then applied at least 3 times daily to the debrided area. Follow-up visits with nail photography were planned every 2 weeks after the procedure to check treatment progress. Complete clinical resolution of the condition was achieved after 5 months (Fig. 2). Ten-month follow-up revealed no recurrence of the condition.

\section{Discussion}

Fungal nail infections are common infections of the fingernails and/or toenails that can cause the nail to become discolored, thick, and more likely to crack and break. Infections are more common in toenails than fingernails [6]. Usually, one or both great toenails are af- 
fected. Dermatophytoma represents a more advanced form of onychomycosis. It appears as linear, single, or multiple white or yellow bands on the nail plate [7]. A dermatophytoma indicates a fungal mass of hyphae and spores within or under the nail plate. The fungi in these masses create a biofilm that this is considered a negative prognostic factor and particularly difficult to treat without debridement [8]. Oral antifungal treatment alone is not enough to treat this condition [9] and either chemical or physical debridement is essential to eliminate dermatophytoma [10].

The case presented herein shows longitudinal band of yellow nail plate discoloration clinically typical with dermatophyoma supported by positive direct microscopy and fungal culture which yield Trichophyton rubrum as the causative agent which is considered the most common etiologic fungus in such condition. Interestingly, the onychomycotic band is coupled with a longitudinal nail splitting of the same length which could be a very rare or 1 st reported association. This splitting might be enhanced by the fungus colony within or underneath the nail plate owing to the keratolytic effect of the dermatophyte. This could be further enhanced by repeated minor trauma to the nail.

Despite the number of available treatments, not all patients having onychomycosis with or without dermatophyoma are cured. Treatment is aimed at eradication of the causative fungus and return to a normal appearance of the nail. Numerous factors have been cited to explain the lack of response to therapy, such as nonadherence to treatment, incorrect diagnosis, or advanced disease. Dermatophytoma, involvement of lateral aspect of the nail, $>50 \%$ of nail affected, "spikes" extending from distal to proximal nail, and subungual hyperkeratosis $>2 \mathrm{~mm}$ are among the factors [11]. Approaches to treat onychomycosis are varied. Lines of therapy can be categorized as topical, oral, or device related. Oral treatment was the mainstay for onychomycosis therapy for long periods. A combination treatment is often the best choice. Topical therapies are generally unsuccessful and have a limited efficacy if used without nail plate debridement. Nail debridement or avulsion can be achieved by the application of urea $40 \%$ under occlusion. Once weekly application of urea $40 \%$ and bifonazole $1 \%$ ointment and sealing resulted in earlier nail removal in onychomycosis [12]. Simultaneous nail debridement further improves treatment outcome by increasing drug efficacy and cure rates.

The water-soluble ciclopirox $8 \%$ nail lacquer formulation has been approved and marketed in $>40$ countries. It does not require nail filing and is easily removed with wa- ter, thereby greatly improving patient compliance [13]. This $8 \%$ formulation is not available in some other countries like Egypt. When used alone, ciclopirox $8 \%$ solution has a mycotic cure rate of $29-36 \%$, and a clinical cure rate of $6-9 \%$ [14]. A Cochrane review noted that the treatment failure rate was $61-64 \%$ after 48 weeks of use [15]. $\mathrm{Wu}$ et al. [16] were the only workers who investigated the efficacy of ciclopirox olamine $1 \%$ cream formulation in 42 onychomycosis patients in a clinical trial in Taiwan. The patients used the cream 2-3 times a day for 3-24 months. In addition, infected nails were filed to facilitate drug penetration. Cure was achieved more easily, if there was only modest alteration of the nail plate and occurred in $14 \%$ of the patients while $36 \%$ improved and $50 \%$ showed no response.

Most studies showed onychomycosis to be highly resistant to topical antifungal therapy. Topical treatment with ciclopirox olamine alone is less effective as it is often associated with poor drug permeation through the nail plate [1] and it has a failure rate exceeding 60\% [11]. Based on these facts, the approach to this patient was to adopt 2 lines of treatment, nail debridement concomitant with topical ciclopirox $1 \%$ solution owing to its availability and absence of $8 \%$ formulation in Egypt. With the combined approach, the nail returns gradually to normal with complete restoration of nail anatomy and an excellent response obtained within 5 months. Being non or minimally invasive, inexpensive technique, does not require special training and can be done in office, this treatment option can be advocated for similar nail conditions avoiding unnecessary and expensive lines of treatment.

\section{Statement of Ethics}

The study was approved by the Institutional Review Board of the Faculty of Medicine and the patient has given her consent to publish photos and details of the case.

\section{Conflict of Interest Statement}

The author has no conflict of interest to disclose.

\section{Funding Sources}

The authors did not receive any funding.
Nail Dermatophytoma Managed with Debridement Plus Local Therapy
Skin Appendage Disord 2021;7:127-130 DOI: $10.1159 / 000512037$ 


\section{References}

1 Hassan N, Dhamija P, Bharti V, Vishwakarma S, Mansoor S, Iqbal Z. Clinical tools for successful treatment of onychomycosis: a narrative review. Drugs Ther Perspect. 2020; 36(6):236-42.

2 Aggarwal R, Targhotra M, Kumar B, Sahoo $\mathrm{PK}$, Chauhan MK. Treatment and management strategies of onychomycosis. J Mycol Med. 2020;30(2):100949.

3 Burkhart CN, Burkhart CG, Gupta AK. Dermatophytoma: recalcitrance to treatment because of existence of fungal biofilm. J Am Acad Dermatol. 2002 Oct;47(4):629-31.

4 Wang C, Cantrell W, Canavan T, Elewski B. Successful treatment of dermatophytomas in 19 patients using efinaconazole $10 \%$ solution. Skin Appendage Disord. 2019;5(5):304-8.

5 Aly R, Winter T, Hall S, Vlahovic T. Topical tavaborole in the treatment of onychomycosis complicated by dermatophytoma: a post-hoc assessment of phase II subjects. J Drugs Dermatol. 2018;17(3):347-54.
6 Gupta AK, Jain HC, Lynde CW, Macdonald P, Cooper EA, Summerbell RC. Prevalence and epidemiology of onychomycosis in patients visiting physicians' offices: a multicenter Canadian survey of 15,000 patientsexternal icon. J Am Acad Dermatol. 2000 Aug; 43(2 Pt 1):244-8.

7 Martinez-Herrera E, Moreno-Coutiño G, Fernández-Martínez RF, Finch J, Arenas R. Dermatophytoma: description of 7 cases. J Am Acad Dermatol. 2012;66(6):1014-6.

8 Freedman JB, Tosti A. Distal subungual onychomycosis. In: Tosti A, Vlahovic TC, Arenas $\mathrm{R}$, editors. Onychomycosis: an illustrated guide to diagnosis and treatment. 1st ed. Springer; 2017. p. 21-34.

9 Sigurgeirsson B. Prognostic factors for cure following treatment of onychomycosis. J Eur Acad Dermatol Venereol. 2010;24(6):679-84.

10 Epstein E. How often does oral treatment of toenail onychomycosis produce a disease-free nail? Arch Dermatol. 1998;134:1551-4.

11 Westerberg DP, Voyack MJ. Onychomycosis: current trends in diagnosis and treatment. Am Fam Physician. 2013 Dec 1;88(11):76270 .
12 Shemer A, Eshel Y, Gupta AK, Farhi R, Haneke E, Daniel CR, et al. Once weekly application of urea $40 \%$ and bifonazole $1 \%$ leads to earlier nail removal in onychomycosis. Skin Appendage Disord. 2020;6(5):304-8..K

13 Iorizzo M, Hartmane I, Derveniece A, Mikazans I. Ciclopirox $8 \% \mathrm{HPCH}$ nail lacquer in the treatment of mild-to-moderate onychomycosis: a randomized, double-blind amorolfine controlled study using a blinded evaluator. Skin Appendage Disord. 2015;1(3):13440.

14 Gupta AK, Fleckman P, Baran R. Ciclopirox nail lacquer topical solution $8 \%$ in the treatment of toenail onychomycosis. J Am Acad Dermatol. 2000;43(4 Suppl):S70-80.

15 Crawford F, Hollis S. Topical treatments for fungal infections of the skin and nails of the foot. Cochrane Database Syst Rev. 2007;3(3): CD001434.

16 Wu YC, Chuan MT, Lü YC. Efficacy of ciclopiroxolamine $1 \%$ cream in onychomycosis and tinea pedis. Mycoses. 1991;34(1-2):93-5. 